\title{
Prognostic impact of perioperative CA19-9 levels in patients with resected perihilar cholangiocarcinoma
}

\author{
Jong Woo LEE ${ }^{1}$, Jae Hoon LEE ${ }^{*, 2}$, Yejong PARK², Woohyung LEE ${ }^{2}$, Jaewoo KWON², Ki Byung SONG ${ }^{2}$, Dae Wook HWANG ${ }^{2}$, Song Cheol KIM² \\ 'Department of Surgery, Hallym University Sacred Heart Hospital, Anyang, Korea \\ ${ }^{2}$ Department of Hepatobiliary and Pancreatic Surgery, Asan Medical Center, University of Ulsan College of Medicine, Seoul, Korea
}

Introduction: The clinical significance of perioperatibe level of carbohydrate antigen (CA) 19-9 in perihilar cholangiocarcinoma (PHCC) has not been well established. This study aimed to examine whether perioperative change of CA19-9 levels could predict prognosis of patients underwent surgery for PHCC.

Methods: This study included 322 patients who underwent curative resection for PHCC. Patients were divided into 3 groups; normal preoperative normal CA19-9 group (CA19-9 $\leq 37 \mathrm{U} / \mathrm{mL}$ ), normalization group (preoperative CA19-9 > 37 U/mL, postoperative CA19$9 \leq 37 \mathrm{U} / \mathrm{mL}$ ) and non-normalization group (both preoperative and postoperative CA19-9 > 37 U/mL). The association of clinicopathological factors (including perioperative serum CA19-9 levels) with overall survival (OS) was investigated.

Results: The non-normalization group (82 patients) showed significantly worse OS than normal CA19-9 group (114) and normalization group (126) (5-year OS, $16.9 \%, 29.4 \%$, and $34.4 \%$; both $p \leq 0.001$ ). Cut-off points of preoperative $300 \mathrm{U} / \mathrm{mL}(p=0.001)$ and postoperative $37 \mathrm{U} / \mathrm{mL}(p<0.001)$ showed highest significant prognostic value. In the non-normalization group, patients who underwent $\mathrm{R} 1$ resection showed significant worse OS than those who underwent R0 resection (median OS, 10.2 vs. 15.7 months; $p=0.016$ ). In multivariable analysis, factors independently associated with worse OS were lymph node metastasis (hazard ratio (HR) $2.07 ; p<0.001$ ), postoperative CA19-9 > $37 \mathrm{U} / \mathrm{mL}$ (HR 1.94; $p<0.001)$, intraoperative transfusion (HR 1.74; $p=0.002)$, advanced T stage (T3, 4) $(\mathrm{HR}$ $1.67 ; p=0.006)$.

Conclusions: Persistent high CA19-9 level after resection of PHCC with curative intent was associated with poor OS. R1 resection was associated with poor OS especially in non-normalization group. High postoperative CA19-9 value was also independent significant prognostic factor in resected PHCC. 